\title{
Distinct Polarization Dynamics of Microglia and Infiltrating Macrophages: A Novel Mechanism of Spinal Cord Ischemia/Reperfusion Injury
}

\author{
Hui Li ${ }^{1,2}$ \\ Pengfei Wang ${ }^{1,2}$ \\ Lin Tang ${ }^{1,2}$ \\ Jingjing Sun ${ }^{1,2}$ \\ Yanling Zhang ${ }^{1,2}$ \\ Wei Luo ${ }^{1,2}$ \\ Cong Luo ${ }^{1,2}$ \\ Zhaolan $\mathrm{Hu}^{1,2}$ \\ Lin Yang (D) ${ }^{1,2}$ \\ 'Department of Anesthesiology, The \\ Second Xiangya Hospital, Central South \\ University, Changsha, People's Republic \\ of China; ${ }^{2}$ Hunan Province Center for \\ Clinical Anesthesia and Anesthesiology, \\ Research Institute of Central South \\ University, Changsha, People's Republic \\ of China
}

Correspondence: Lin Yang Department of Anesthesiology, The Second Xiangya Hospital, Central South University, Changsha, People's Republic of China

Fax +86 73185295970

Email linyang@csu.edu.cn
Purpose: Recent studies indicate that microglia and monocyte-derived macrophages (MDMs) have different roles in diseases such as stroke and spinal cord injury, yet their respective polarized phenotypes and roles remain unclear in spinal cord ischemia/reperfusion injury (SCIRI).

Methods: We established a mouse model of SCIRI by transient aortic occlusion followed by reperfusion. Basso mouse scale (BMS) scores were used to test the locomotor functions. The histopathological changes in spinal cord were assessed by hematoxylin-eosin staining and NF-200 immunohistochemistry. Real-time PCR, immunofluorescence and flow cytometry were employed to analyze the polarized phenotypes of the microglia and infiltrating MDMs, and the resulting inflammatory responses. Furthermore, the role of infiltrating MDMs were investigated by MDMs depletion using systemic administration of clodronate-liposomes.

Results: SCIRI significantly impaired locomotor function of mice, accompanied with progressed necrosis, infiltration of inflammatory cells and neuron loss in the spinal cord. M1-related proinflammatory markers (iNOS, CD16, CD86 and TNF- $\alpha$ ) increased dramatically in the early phase following SCIRI. In contrast, M2-related anti-inflammatory markers (CD204 and CD206) elevated at later stage. Besides, the invading MDMs were principally pro-inflammatory M1 type, transiently restricted to the first week after SCIRI. In contrast, microglia were the main source of antiinflammatory M2 type. Furthermore, depletion of MDMs by clodronate-liposomes significantly preserved neurological functions and relieved neuronal damage caused by SCIRI.

Conclusion: These findings suggested distinct polarized status of resident microglia and MDMs following SCIRI. Inhibition of the invading MDMs may represent a novel approach for SCIRI treatment.

Keywords: spinal cord ischemia/reperfusion injury, microglia, monocyte derived macrophages, polarization, inflammation

\section{Introduction}

Spinal cord ischemic/reperfusion injury (SCIRI) remains a devastating complication after thoracoabdominal aortic surgery. ${ }^{1}$ Conventional strategies, including hypothermic circulatory arrest and cerebrospinal fluid drainage, do not provide sufficient protection for the prevention of SCIRI-induced paraplegia. ${ }^{2}$ Once the injury has manifested clinically, no pharmacological or surgical intervention is currently available. Therefore, new treatment strategies targeting different stages of SCIRI are urgently required.

A growing body of evidence has revealed that resident microglia and infiltrating monocyte-derived macrophages (MDMs) are the primary immune cells and have been widely targeted to improve outcomes after ischemic injury of the central nervous system 
(CNS). ${ }^{3,4}$ Despite sharing the same cell markers and similar morphology, microglia and MDMs can display distinct functions through polarization in response to special environmental stimuli. ${ }^{5,6}$ Previous studies have shown that microglia produce high levels of IL-10 and display greater myelin phagocytosis capability compared with MDMs, indicating that microglia are more likely to be the source of this anti-inflammatory M2-type cytokine in inflammatory conditions. ${ }^{7-9}$ In terms of MDMs, some studies support a detrimental role and have established that infiltrating MDMs could aggravate the inflammation response, therefore exacerbating neuron damage in the $\mathrm{CNS} ;{ }^{7-11}$ While others argue that infiltrating MDMs are vital cells that exert anti-inflammatory properties and improve the recovery from brain and spinal cord injury. ${ }^{12-15}$ Therefore, the dynamics of infiltration and the polarized status of MDMs and microglia appear to be critical for determining neurological outcome. However, evidences in relation to their roles and polarized status in the pathological processes occurring in SCIRI remain lacking.

In the present study, a well-established murine model of SCIRI was used to determine the precise activation and polarization patterns of microglia and MDMs over time following injury. We demonstrated for the first time that resident microglia and infiltrating MDMs initially exhibited an M1 phenotype in the early stages of ischemia-reperfusion, gradually switching to an M2 phenotype and remaining for a later period of time. Furthermore, we also determined that MDMs recruited from the peripheral circulation were primarily characterized by the M1 type, which might worsen early neurological disability after SCIRI.

\section{Materials and Methods}

\section{Mice}

Adult male C57BL/6 mice, aged 12 weeks, were purchased from Silaike Jingda Experimental Animal Co., Ltd (Changsha, Hunan, China) and were provided with food and water ad libitum. All procedures were performed using aseptic techniques and approved by the Institutional Animal Care and Use Committee of Central South University and conducted according to the Health Guidelines of the National Institutes for the Use and Care of Laboratory Animals.

\section{Animal Grouping}

In order to detect the polarized status of microglia and macrophage after SCIRI takes place, 84 mice were randomly divided into two groups: the Sham group and the
SCIRI group. Furthermore, in order to investigate the effect of infiltrating MDMs on SCIRI, another 81 mice were randomly divided into four groups based on using either clodronate liposomes to deplete the monocytes or empty liposomes as a negative control: the Sham+CloLip group, the Sham+EmpLip group, the SCIRI+CloLip group and the SCIRI+EmpLip group.

\section{Descending Aorta Clamping}

Mice were anesthetized with intraperitoneal injection (i.p.) of pentobarbital $(50 \mathrm{mg} / \mathrm{kg})$, and were subjected to a moderate SCIRI established by descending aorta clamping (DAC), as described by a previous research. ${ }^{16}$ Briefly, mice received tracheal intubation using a 1.5-inch, 20-gauge IV catheter (BD Insylte, Sparks, MD) as described in detail in a previous study. ${ }^{17}$ Ventilation (SAR-1000, CWE Inc, PA) was set with the following parameters: concentration of oxygen at $50 \%$, tidal volume at $400 \mu \mathrm{L}$ and rate at $230 \mathrm{bpm}$. Next, a small transverse incision (dorsal to ventral, beneath the 2nd rib) was created below the left forelimb and shoulder; and the descending aorta, beginning $1 \mathrm{~mm}$ distal to the left subclavian artery, was exposed and completely cross-clamped with aneurysm clips for $9 \mathrm{~min}$. After that, the chest was closed and the animals ventilated until recovery had been achieved. In contrast, mice in the Sham group only had the aortic arch exposed without DAC.

During the surgical procedure, the core temperature of the animals was maintained at $37^{\circ} \mathrm{C}$ using a temperaturecontrolled operating bed. Left femoral mean blood pressure (FMBP) was monitored and samples for blood-gas analysis were collected $5 \mathrm{~min}$ prior to ischemia and $10 \mathrm{~min}$ after reperfusion.

\section{MDMs Depletion}

At a half hour post DAC, mice in the SCIRI group received intraperitoneal bolus dose of either clodronate liposomes $(200 \mu \mathrm{L}, 7 \mathrm{mg} / \mathrm{mL}$, SCIRI+CloLip group, FormuMax, CA, US) or empty liposomes $(200 \mu \mathrm{L}, 7 \mathrm{mg} /$ $\mathrm{mL}$, SCIRI+EmpLip group, FormuMax), followed by $100 \mu \mathrm{L}$ respectively every three days according to the manufacturer's instruction. As reference, mice in the Sham group also received equal volume of either clodronate liposomes (Sham+CloLip group) or empty liposomes (Sham+EmpLip group) at the same time points.

\section{Functional Locomotor Scores}

As performed in a previous study, ${ }^{18}$ Basso mouse scale (BMS) scores, ranging from 0 (totally paraplegia) to 9 (normal) were 
assessed to quantify hind limb function in mice of each group at postoperative day 1 (P1), P3, P5, P7 and P14 following reperfusion ( $\mathrm{n}=9$ for each group). Assessments were conducted by two researchers blinded to the study grouping, and the average values were used for the final results.

\section{Histological Analysis}

To detect the morphological changes of spinal cord following SCIRI, 27 mice were placed into deep anesthesia using an overdose of sevoflurane. Then, trans-cardiac perfusion of $150 \mathrm{~mL}$ of cold PBS containing $0.1 \%$ heparin was performed, followed by $500 \mathrm{~mL}$ of ice-cold $4 \%$ paraformaldehyde. After that, lumbar enlargements of spinal cord were harvested, fixed in 4\% paraformaldehyde for 8 hours, then cryoprotected in $30 \%(\mathrm{w} / \mathrm{v})$ sucrose. Serial frozen sections with $10 \mu \mathrm{m}$-thickness were created then stained with hematoxylin-eosin (HE) method.

\section{Immunohistochemistry and Immunofluorescence}

To further analyze the neuron loss and expression changes of M1/M2 markers for macrophage/microglia polarization, the acquired frozen sections were further analyzed with immunohistochemistry and immunofluorescence, which have been described in our previous research. ${ }^{19}$ Briefly, for immunohistochemistry, rabbit anti-mouse NF-200 (1:500, Proteintech, IL, US) was used as the primary antibody to mark neuron, followed with Biotin-conjugated goat anti-rabbit $\operatorname{IgG}(1: 100$, Proteintech, IL, US) as the secondary antibody. For immunofluorescence, primary antibodies included rabbit anti-mouse CD11b (1:100, Affinity, OH, US) to mark macrophage/ microglia, rat anti-mouse CD86 (a recognized M1 marker, 1:100, R\&D system, MN, US), and mouse anti-mouse CD206 (a recognized M2 marker, 1:100, Abcam,
Cambridge, UK), followed with secondary antibodies including donkey anti-rabbit, donkey anti-mouse and donkey antirat IgG (1:500, Jackson Immuno-Research, PA, US). All sections were imaged microscopically (Olympus U-HGLGPS, Japan) and analyzed using ImageJ software (National Institutes of Health) for positive cell counting.

\section{Real-Time PCR}

To seek the exact expression patterns of M1/M2 polarizations of macrophage and microglia, 15 mice were euthanized using an overdose of sevoflurane and their spinal cords harvested on ice. Total RNA was extracted using Trizol $^{\mathrm{TM}}$ reagent (Thermo Fisher Scientific, MA, US). cDNA for each group was synthesized by reverse transcription of the RNA (Thermo Fisher Scientific, MA, US). Real-time PCR was performed using a CFX96 Touch $^{\mathrm{TM}}$ Deep Well Real-Time PCR Detection System (Bio-Rad Laboratories, CA, US) and $S Y B R^{\circledR}$ Green Real-Time PCR Master Mix (Roche, Germany). The primers are listed in Table 1. Quantitative PCR was performed as described in a previous study ${ }^{20}$ and repeated in triplicate. Data were processed using the $2^{-\Delta \Delta \mathrm{Ct}}$ method.

\section{Flow Cytometric Analysis}

To investigate the dynamic changes of microglia/macrophage polarization and the effect of monocyte depletion on infiltrating MDMs after SCIRI, the spinal cords of 81 mice were harvested, perfused with PBS and immediately filtered through a $40 \mu \mathrm{m}$ nylon cell strainer (BD Biosciences, NY, US) in order to separate cells from myelin and debris with Percoll density gradient medium (GE Healthcare; 70\% and 30\%). The cells were carefully collected from the interface of the gradient, washed, then stained for flow cytometric analysis using the following antibodies or the respective isotype controls for 20

Table I Primers of Genes Used in PCR Analysis

\begin{tabular}{|l|l|l|}
\hline Primer & \multicolumn{1}{|c|}{ Forward } & \multicolumn{1}{|c|}{ Reverse } \\
\hline iNOS & GAACGGAGAACGTTGGATTTG & TCAGGTCACTTTGGTAGGATTT \\
TNF- $\alpha$ & TTGCTCTGTGAAGGGAATGG & GGCTCTGAGGAGTAGACAATAAAG \\
CDI6 & CATCAGCTCCTGTCTGGTTT & CTCTCTGCAGCCTGTGTATTT \\
CD86 & TGGTTCTGTACGAGCACTATTT & GTAGAGTCCAGTTGTTCCTGTC \\
Arg-I & TGGTGTGGTGGCAGA GGTCCA & ACTGCCAGACTGTGGTCTCCACC \\
IL-4 & TGTCATCCTGCTCTTCTTTCTC & TCTGTGGTGTTCTTCGTTGC \\
CD204 & CTGAATGTCAGAGTCCGTGAA & CAGACTGGACTTCTGCTGATAC \\
CD206 & GTGGTCCTCCTGATTGTGATAG & CACTTGTTCCTGGACTCAGATTA \\
GAPDH & GCATCCTGGGCTACACTGAG & TGGTCCTCAGTGTAGCCCAAG \\
\hline
\end{tabular}

Abbreviations: iNOS, inducible NO synthase; TNF- $\alpha$, Tumor necrosis factor alpha; Arg-I, arginase-I; IL-4, interleukin 4; GAPDH, glyceraldehyde-3-phosphate dehydrogenase. 
Table 2 Distal Hemodynamics and Blood-Gas Analysis Before and After DAC

\begin{tabular}{|l|c|c|c|}
\hline & Pre-Ischemia & Ischemia & Reperfusion \\
\hline FMBP $(\mathrm{mmHg})$ & $35.8 \pm 2.5$ & $15.8 \pm 5.6^{* *}$ & $32.8 \pm 2.9$ \\
$\mathrm{pH}$ & $7.51 \pm 0.04$ & $\mathrm{Nd}$ & $7.21 \pm 0.08^{* *}$ \\
$\mathrm{PaO}_{2}(\mathrm{mmHg})$ & $91.6 \pm 3.4$ & $\mathrm{Nd}$ & $82.6 \pm 4.2^{*}$ \\
$\mathrm{PaCO}_{2}(\mathrm{mmHg})$ & $33.2 \pm 5.6$ & $\mathrm{Nd}$ & $35 \pm 5.0$ \\
Base Excess & $-2.4 \pm 3.3$ & $\mathrm{Nd}$ & $-11.6 \pm 3.8^{*}$ \\
\hline
\end{tabular}

Notes: Data presented as mean \pm standard deviation. $*$ and $* *$ separately represent $P<0.05$ and $P<0.01$ vs pre-ischemic values.

Abbreviation: FMBP, Left femoral mean blood pressure.

min on ice: PE-cy7-labeled rat anti-mouse CD11b (1:100, BioLegend, CA, US), FITC-labeled rat anti-mouse CD45 (1:100, BioLegend), APC-labeled rat anti-mouse CD369 (1:100, BioLegend) or BV421-labeled rat anti-mouse CD86 (1:100, BioLegend). To determine the absolute numbers of cells, the cell concentration was calculated with Precision Count Beads (BioLegend, San Diego, CA, USA) according to manufacturer's protocol. The cells were finally analyzed on the flow cytometer (Cystek, Fremont, CA, USA) using the FlowJo V10.0 software (FlowJo, OR, US).

To determine the effect of monocyte depletion in blood, 12 mice were euthanized by isoflurane overdose at P3 after the second shot of clodronate or empty liposomes were administrated. After red blood cells were removed with the application of $\mathrm{NH}_{4} \mathrm{Cl}$ lysis buffer, the single-cell suspensions from spleen were obtained by mechanical disruption and analyzed using the following antibodies: PE-cy7-labeled rat anti-mouse CD11b (1:100, BioLegend) and PE-labeled rat anti-mouse F4/80 (BioLegend, 1:100). The cells were finally analyzed on the flow cytometer using the FlowJo V10.0 software.

\section{Statistical Analysis}

All data were analyzed using SPSS v19.0 (IBM, NY, US) and charted with Prism v8.0 (GraphPad Software, CA, US). Differences were evaluated using one-way ANOVA with individual $t$ test post hoc analysis if data were normally distributed or by Kruskal-Wallis testing with individual Mann-Whitney $U$-test post hoc analysis otherwise. Results would be considered statistically significant when $P<0.05$.

\section{Results}

\section{SCIRI Causes Acute Paraplegia, Spinal} Morphological Change and Neuron Loss

Physiological parameters including distal hemodynamics and blood-gas analysis are presented in Table 2. Detailly, compared with the Sham group, FMBP in the SCIRI group fell dramatically during the period of aortic clamping $(15.8 \pm 5.6$ vs $35.8 \pm 2.5 \mathrm{mmHg}, P<0.01)$ and returned to pre-ischemic levels following reperfusion $(32.8 \pm 2.9$ vs $35.8 \pm 2.5 \mathrm{mmHg}$, $P>0.05)$. In addition, reductions in $\mathrm{pH}(7.21 \pm 0.08$ vs $7.51 \pm$ $0.04, P<0.01), \mathrm{PaO} 2(82.6 \pm 4.2$ vs $91.6 \pm 3.4 \mathrm{mmHg}, P<$ $0.05)$, and base excess (BE) $(-11.6 \pm 3.8$ vs $-2.4 \pm 3.3, P<$ 0.05 ) were observed $10 \mathrm{~min}$ after reperfusion compared with 5 min prior to DAC, indicating a successful implementation of aorta clamping.

The time course of neurological function as assessed by BMS scores over the 21 days following reperfusion (Figure 1A). In detail, the BMS scores of the mice in the Sham group remained at the baseline of 9 (normal), while mice subjected to SCIRI achieved lower BMS scores immediately at $\mathrm{P} 1(P<0.01)$, lasted until $\mathrm{P} 7(P<0.01)$, and gradually improved since P14 $(P<0.05$, compared with P1, P3 and P5 in the SCIRI group), which were still lower than the Sham group $(P<0.01)$. The results indicated that DAC for 9 min produced acute and moderate paraplegia.

As displayed in Figure 1C, HE staining of spinal tissue showed that subtle changes became evident on $\mathrm{P} 1$ after DAC, characterized by isolated vacuolar necrosis predominantly in the region of gray matter. Then, necrosis progressed at P3 and P7 following SCIRI, accompanied with increasing infiltration of inflammatory cells. At P14, the inflammatory cells infiltration appeared gradually dissipated. Consistently, a progressive loss of anterior horn motor neurons was detected by NF-200 staining during the two weeks after SCIRI. The pyknosis was apparent at P1, progressed at P3 and P7, and prolonged to P14 $(P<0.01)$ (Figure 1B and $\mathrm{C}$ ).

\section{Time Course of Messenger RNA Expression of $\mathrm{MI}$ and $\mathrm{M} 2$ Polarized Markers Following SCIRI}

As shown in Figure 2, compared with the Sham group, expression of the M1-type genes in the SCIRI group increased 
A

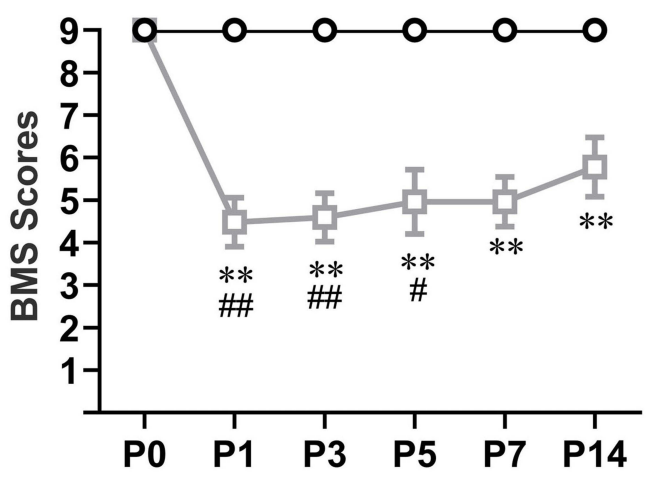

C

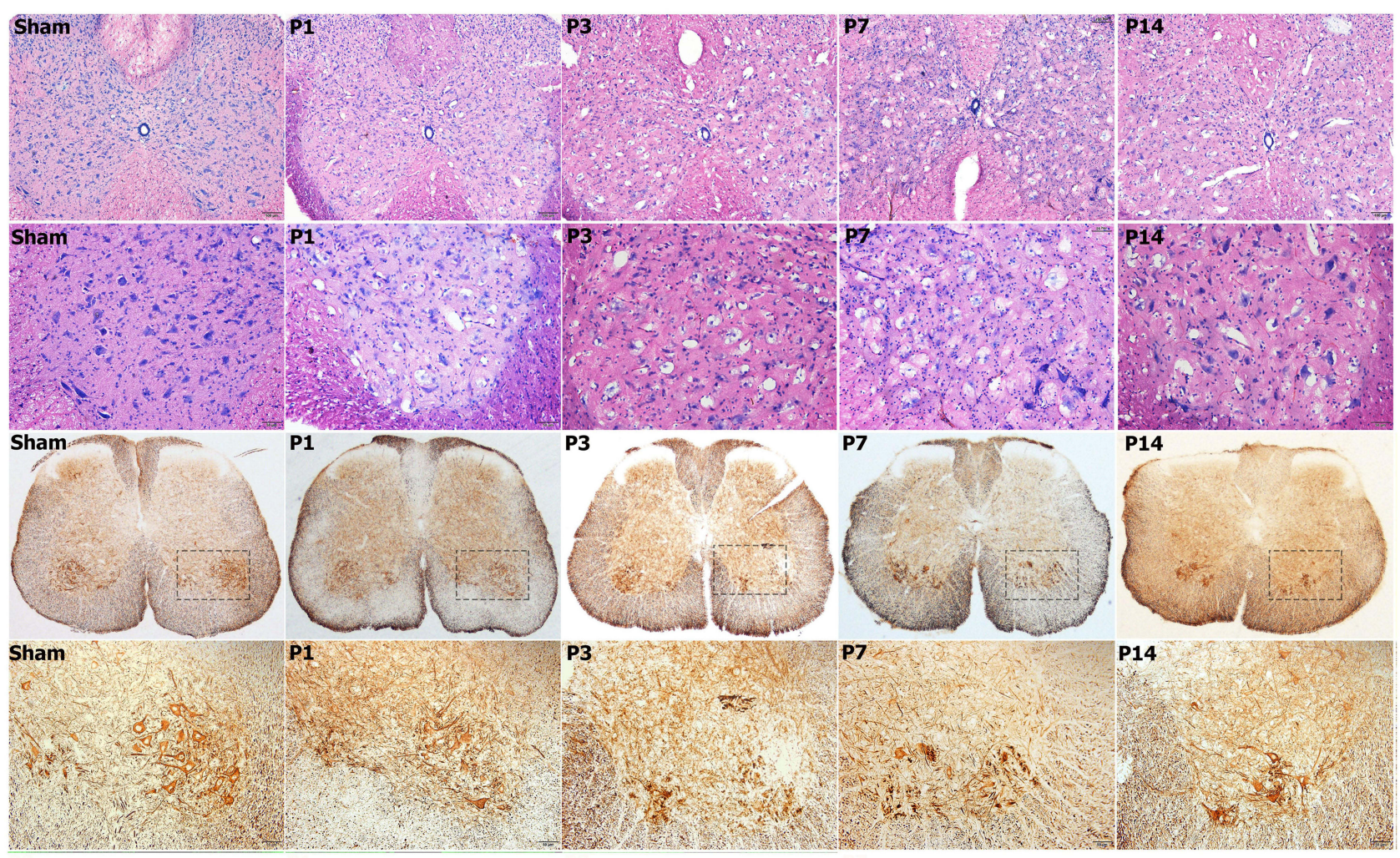

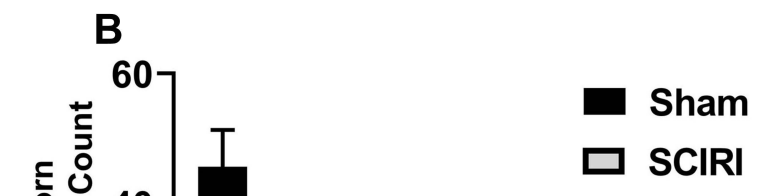

SCIRI
Sham 

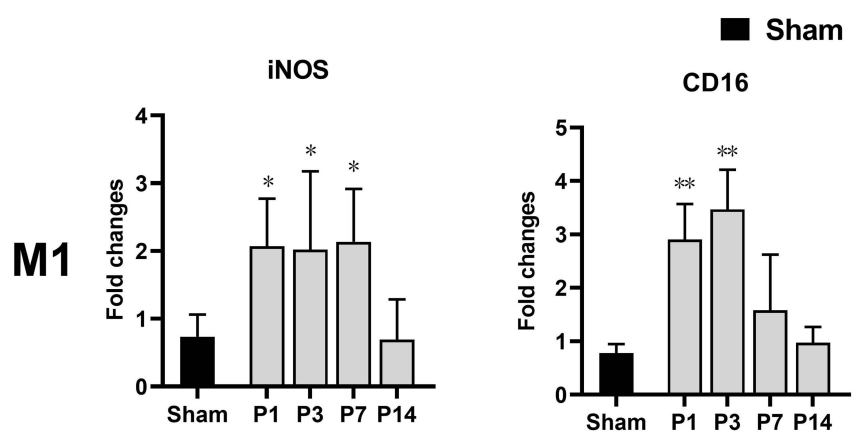

SCIRI

CD86
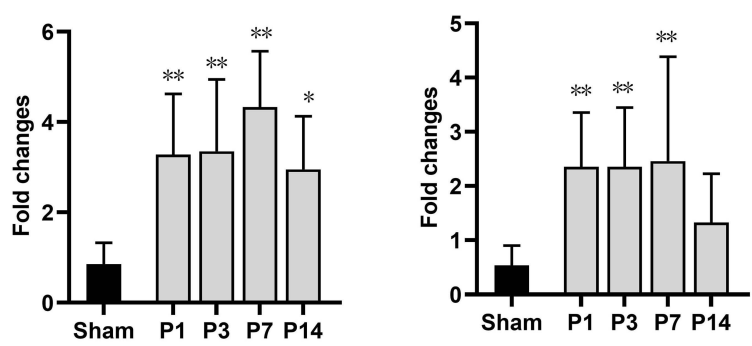

IL-4

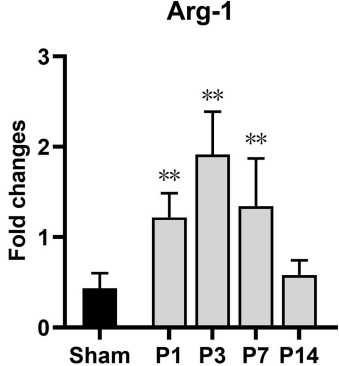

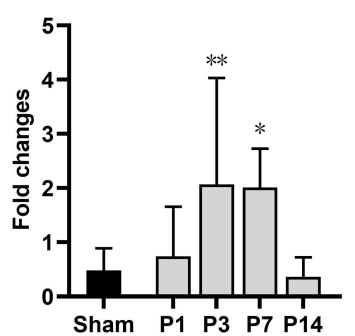

Arg-1

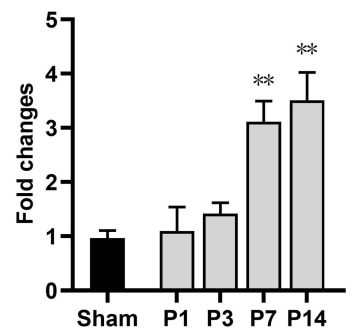

Figure 2 Time course of gene expression of MI and M2 polarization markers following SCIRI. Quantitative PCR was used to assess the expression levels of MI- and M2-like microglial/macrophage activation genes in spinal cord of mice following SCIRI. $n=5$ per group at each time point; $* P<0.05$, $* * P<0.01$ compared with the Sham group. Abbreviation: SCIRI, spinal cord ischemia/reperfusion injury.

In contrast, expression of the M2-type genes including CD204 and CD206 remained at the Sham levels for the first week $(P>0.05)$, then started to increase at P7 (CD204: 3.00fold, $P<0.01$; CD206: 3.22-fold, $P<0.01)$ and remained at a high level at P14 (CD204: 5.28-fold, $P<0.01$; CD206: 3.63-fold, $P<0.01$ ). Instead, other M2 genes including IL-4 and Arg-1 increased at P3 and P1 respectively (IL-4 P3: 4.30-fold, $P<0.01$; Arg-1 P1: 2.79-fold, $P<0.01$ ), remained at a high level for the first week (P7, IL-4:4.17-fold, $P<$ 0.05, $P=0.01$; Arg-1: 3.08-fold, $P<0.01)$, then both returned to preinjury level at $\mathrm{P} 14(P>0.05)$.

\section{Dynamic Changes in Microglia/ Macrophages with $\mathrm{Ml}$ or M2 Phenotypes Following SCIRI}

As shown in Figure 3, the expressions of CD11b and CD86 increased dramatically at P1 $(P<0.01)$ and remained at a high level for a whole week $(P<0.05)$ compared with the Sham group. In contrast, the expression of CD206 stayed unchanged at the early stage (P1 and P3, $P>0.05)$, then increased significantly at $\mathrm{P} 7(P<0.01)$ following SCIRI. Furthermore, double immunofluorescence labeling showed that the expressions of both CD86 and CD206 were predominantly accumulated in the
$\mathrm{CD} 11 \mathrm{~b}^{+}$cells (Figure 4 ). These findings suggested that the M1-type microglia/macrophages would dominate at the early stage of SCIRI, whereas the M2-type would be increasing and occupy the majority at the later stage.

\section{Microglia and Infiltrating MDMs Display Distinct Polarization Patterns During SCIRI}

Here, we used CD11b and CD45 antibodies to distinguish MDMs $\left(\mathrm{CD} 45^{\text {high }} \mathrm{CD} 11 \mathrm{~b}^{+}\right)$from resident microglia $\left(\mathrm{CD} 45^{\text {low }} \mathrm{CD} 11 \mathrm{~b}^{+}\right)$based on previous researches. ${ }^{21-28} \mathrm{As}$ displayed in Figure $5 \mathrm{~A}$ and $\mathrm{B}$, gating of the $\mathrm{CD} 45^{\text {high }}$ populations revealed that infiltrating MDMs increased rapidly and robustly since $\mathrm{P} 3$ post-injury $(P<0.01)$, peaked at $\mathrm{P} 7(P<0.01)$ and fell to the Sham levels since P14 $(P>0.05)$. Furthermore, MDMs expressing CD86 increased consistently and sharply from $\mathrm{P} 3$ to $\mathrm{P} 7$ post SCIRI injury $(P<0.01$, Figure 5C), whereas MDM expressing Dectin remained unchanged at each timepoint ( $P>0.05$, vs Sham group, Figure 5D). In contrast, resident microglia expressing CD86 increased at P3 post-injury $\left(P=0.022\right.$, Figure 5E), while Dectin ${ }^{+}$microglia increased at P7 after SCIRI $(P=0.014$, Figure 5F). 
A

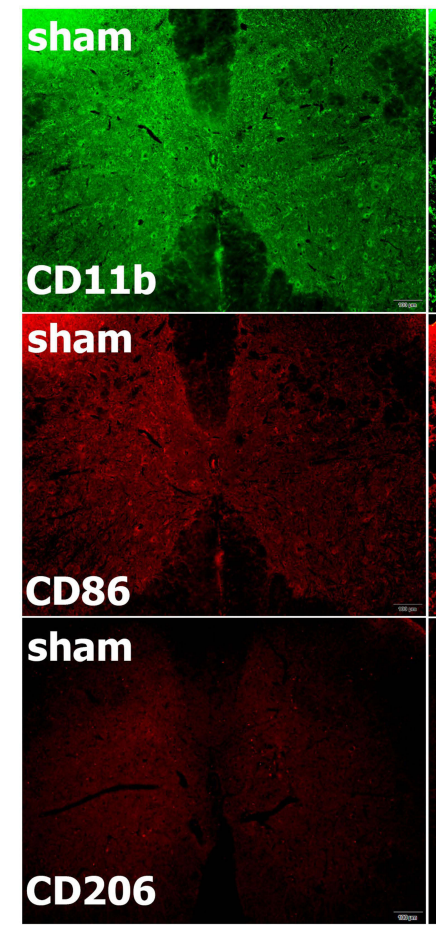

B

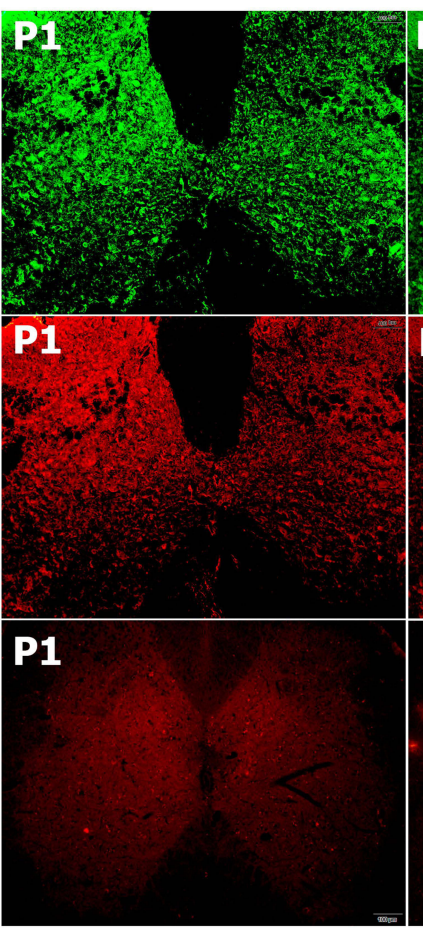

Sham
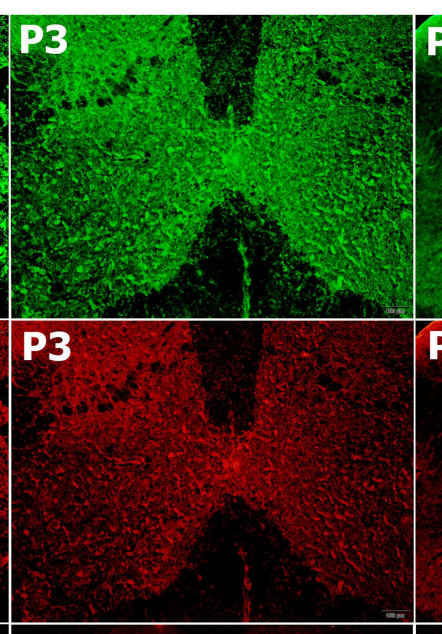

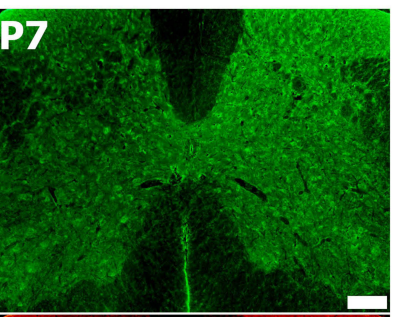

P7

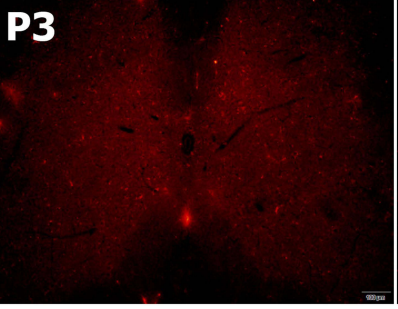

SCIRI

\section{CD11b}
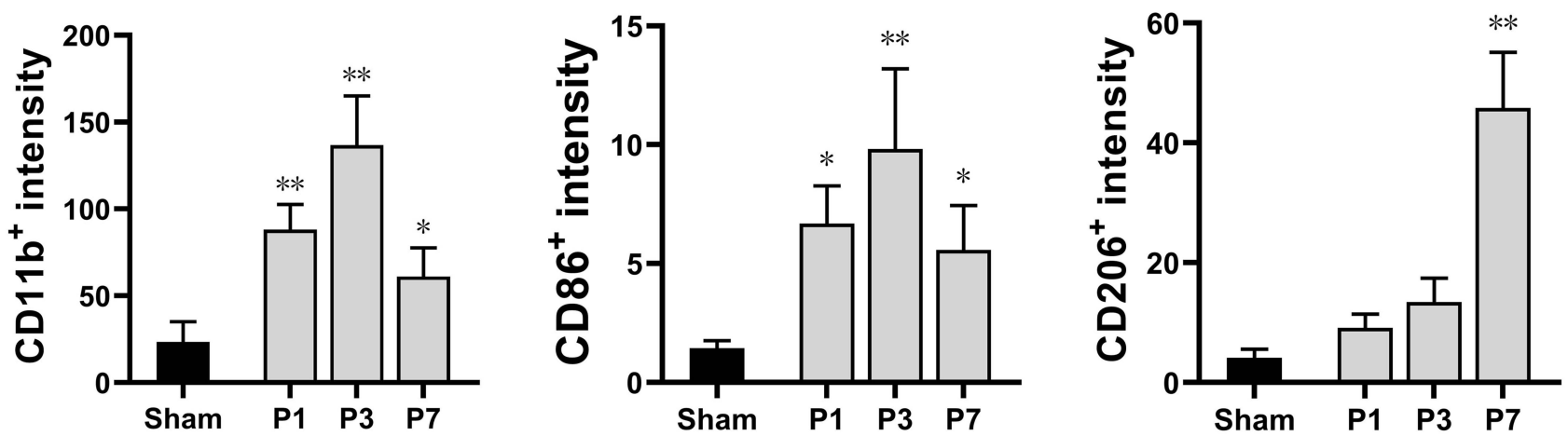

Figure 3 Dynamic changes in MI and M2 phenotypes of macrophages following SCIRI. (A) The immuno-fluorescence staining of CDI Ib ${ }^{+}$(green), CD86 ${ }^{+}$(red) and CD206 ${ }^{+}$ (red) at PI, P3 and P7 after SCIRI. Scale bar $=100 \mu \mathrm{m}, \mathrm{n}=3$ for each time point. (B) Quantification of CDI I b ${ }^{+} \mathrm{CD} 6^{+}$and $\mathrm{CD} 206^{+}$expressions at PI, P3 and P7 after SCIRI. *Represented $P<0.05$ compared with the Sham group. **Represented $P<0.01$ compared with the Sham group.

Abbreviation: SCIRI, spinal cord ischemia/reperfusion injury.

\section{Depletion of MDMs Improves Long-Term Functional Recovery and Spinal Cord Damage}

Considering infiltrating MDMs were mainly proinflammatory M1 subtype, we further determined whether depletion of infiltrating MDMs could improve neurological outcomes following SCIRI. The depletion of MDMs was confirmed by cell counts, while neutrophil was not affected (Figure 6A and B). Consistently, MDMs infiltration to spinal cord was significantly alleviated after clodronate liposomes applications at P3 following SCIRI $(P<0.01)$ (Figure 6C). Besides, compared with SCIRI+EmpLip group, mice in the SCIRI+CloLip group showed a spontaneous improvement in locomotor recovery based upon the BMS score from P3 to P14 $(P<0.01)$ (Figure 6D), accompanied with alleviated vacuolar necrosis and decreased neurons loss in the spinal cord $(P<0.01$, shown in Figure 6E and F). 


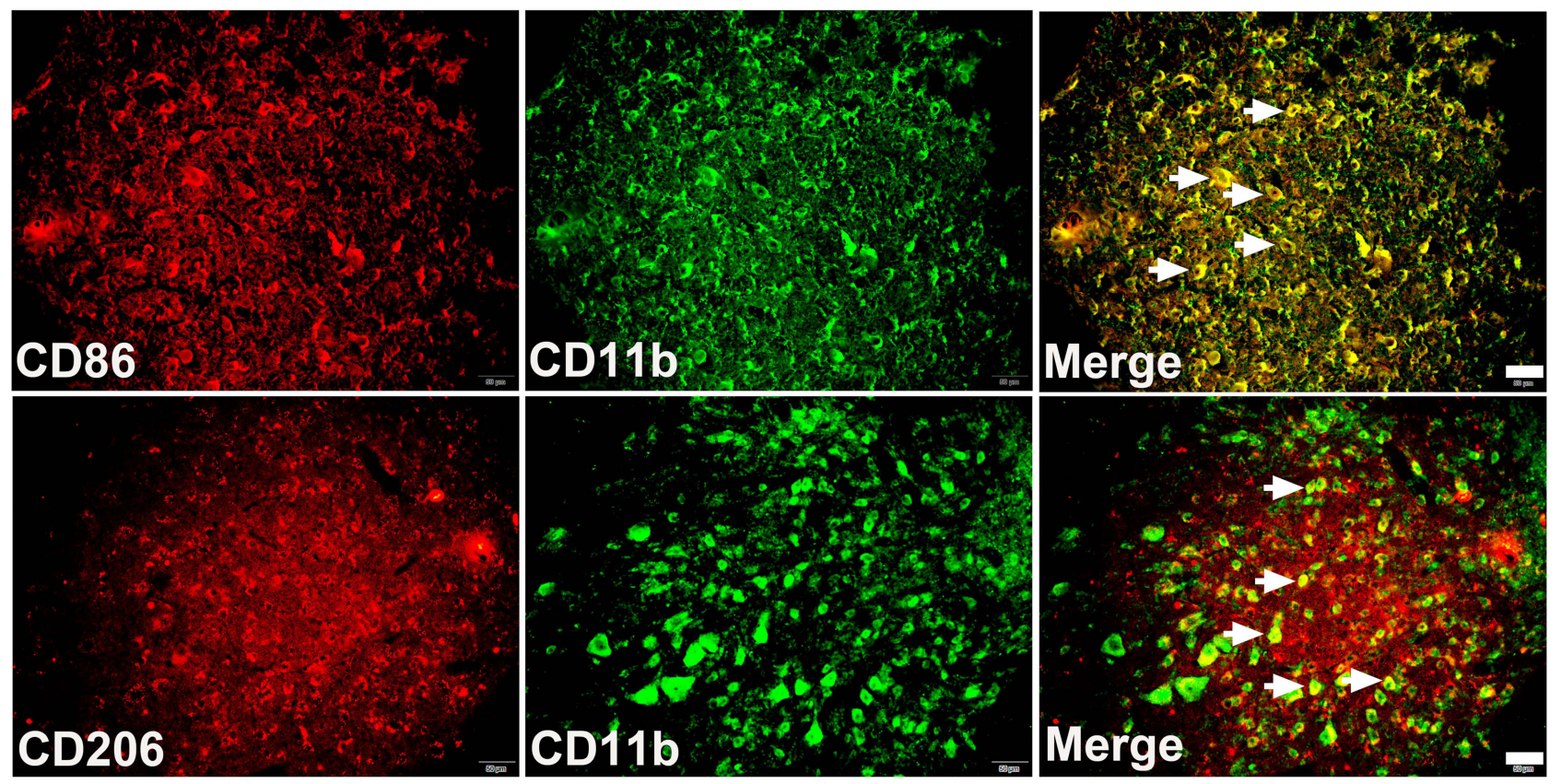

Figure 4 Colocalization of CDIIb and CD86 (or CD206). Double immunofluorescence confirmed that both CD86 (red) and CD206 (red) were mainly accumulated in CDIlb (green) positive cells. scale bar $=25 \mu \mathrm{m}$.

\section{Discussion}

The present study characterized the polarization dynamics of both resident microglia and infiltrating MDMs during SCIRI. The influx of MDMs into spinal cord was transiently restricted to the first week and were primarily proinflammatory M1 type, whereas microglia were principal source of M2 polarized cells at a later stage. Furthermore, we showed for the first time that depletion of infiltrating MDMs markedly alleviated neurological and histological outcomes following SCIRI.

The relative secretion levels of pro- and antiinflammatory factors provide an indication of the polarization state of microglia/MDMs. Thus, we investigated the expression of inflammatory markers at the transcriptional level. Similar with traumatic brain injury, ${ }^{29}$ we observed a sharp upregulation of M1-type genes, such as iNOS, TNF- $\alpha$, CD86, and CD16 following SCIRI, which appeared at an early stage within the 1st week. M1-type cells are believed to release detrimental pro-inflammatory mediators (eg, TNF- $\alpha$, IL-1 $\beta$, and IL-6) and oxidative metabolites, which impair axonal regrowth. ${ }^{5}$ In contrast, M2 biomarkers, including CD204 and CD206, elevated at later stage, mainly after the 1st week. The recruitment of M2 microglia and MDMs may represent an endogenous process aimed at restricting ischemic damage by releasing protective and neurotrophic factors, scavenging cell debris, and resolving local inflammation. ${ }^{5}$
We also noticed that the expressions of Arg1 and IL-4 altered in an uncoordinated manner. Previous studies ${ }^{15,30,31}$ suggested that inflammatory microenvironments may favor a particular biomarker but not others. Of note, $\operatorname{Arg} 1^{+}$macrophages were reported not identical to M2 cells. ${ }^{29}$ Previous studies demonstrated that $\operatorname{Arg} 1^{+}$macrophages also expressed some M1-associated genes like NOS2. ${ }^{32}$ By contrast, Arg $1^{-}$ macrophages could display increased expression of M2 markers. ${ }^{33}$ Therefore, Arg1 might not be used as a special M2 marker in isolation depending on circumstances. In addition, IL-4, known as a T helper type 2 cytokine, has been demonstrated to be a M2 polarization promotor. ${ }^{34}$ Thus, we considered advanced IL-4 elevation might contribute to subsequent M2 macrophage polarization following SCIRI. In the present study, the M1 to M2 conversion occurred 7 days post-injury, coincident with improved neurological and histological outcomes. Interestingly, this polarization conversion is quite different from contusive spinal cord injury, indicating diverse pathological mechanisms underlying these two types of damages to spinal cord.

Peripheral monocytes are known to enter the CNS following injury and play a role in multiple mice models of spinal cord injury. ${ }^{35,36}$ Monocyte infiltration occurs at day 1 or day 3 after the onset of status epilepticus; while in stroke and experimental autoimmune encephalomyelitis (EAE), the initiation of MDMs influx takes place at day $3 .{ }^{37}$ In the present study, we also found that 


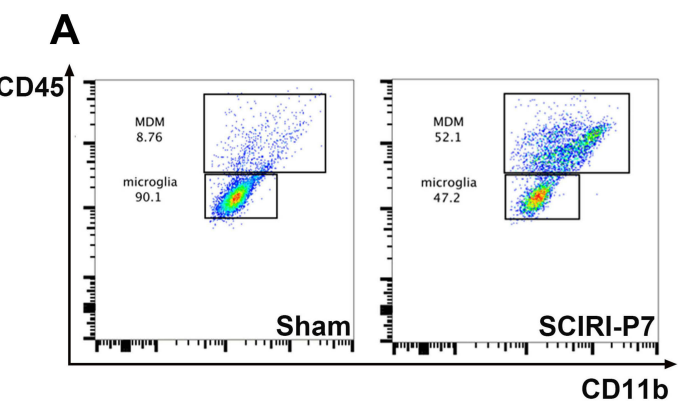

B
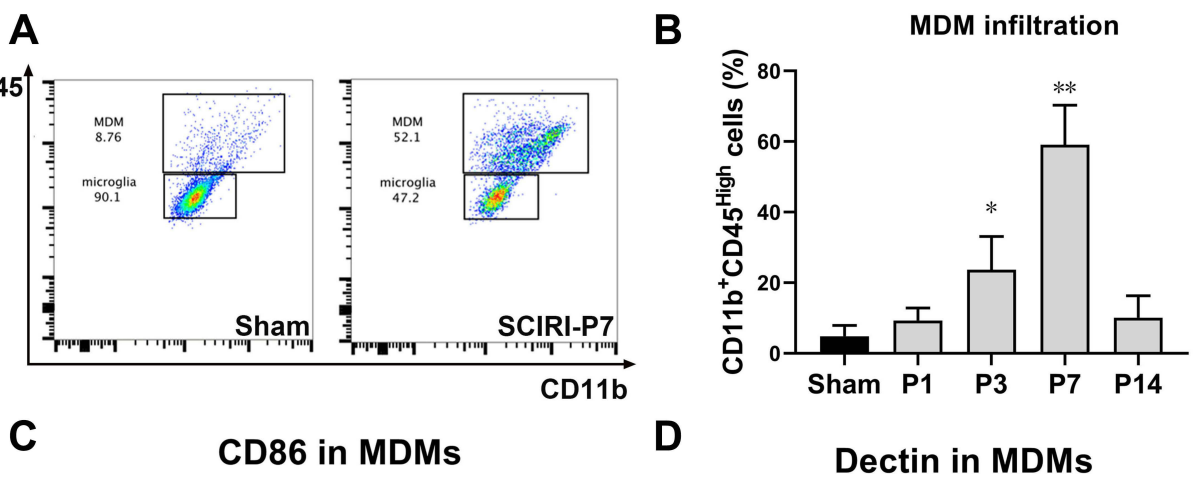

Dectin in MDMs
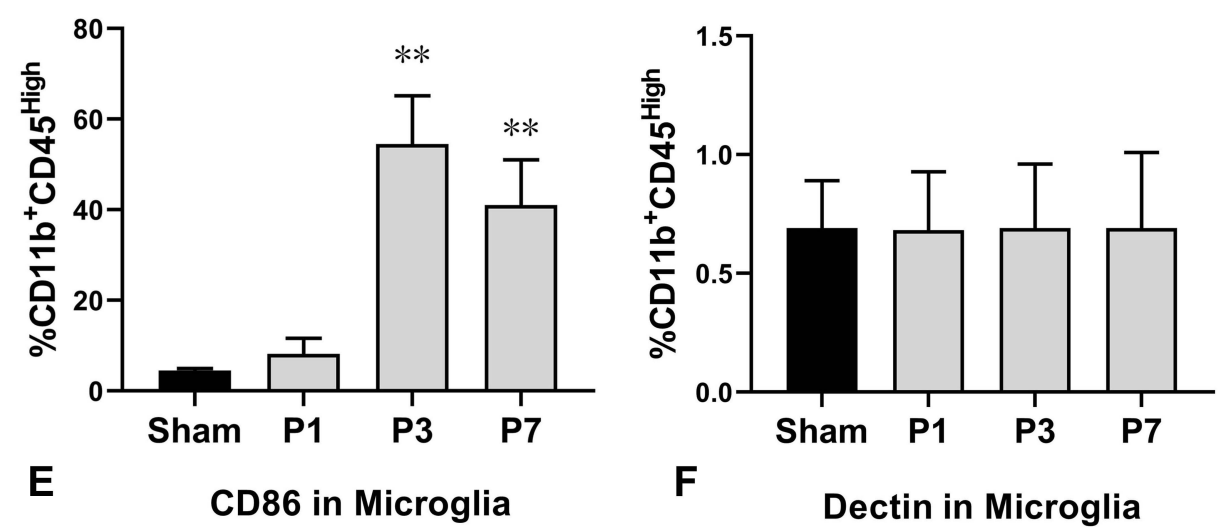

$\mathbf{F}$
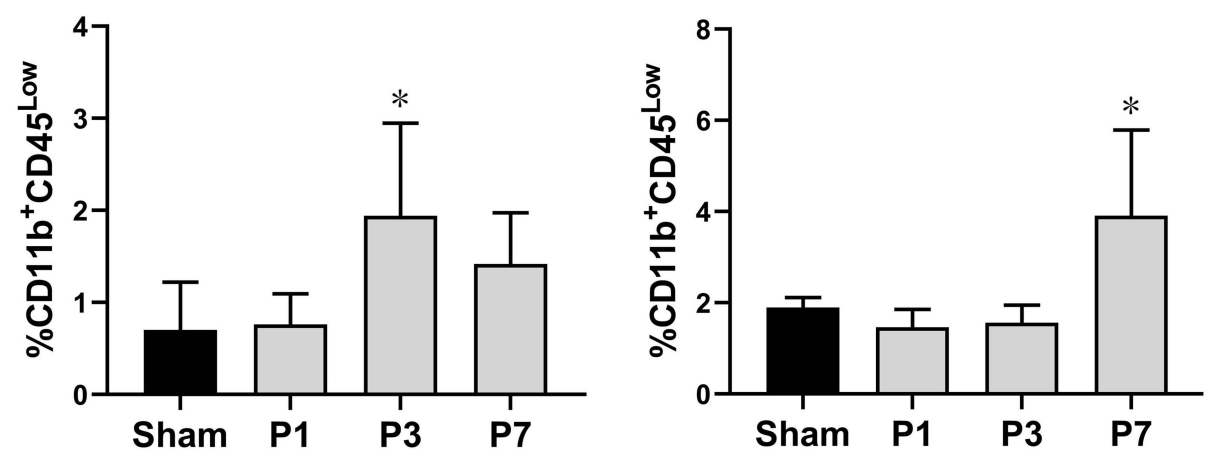

Figure 5 Microglia and invading MDMs display distinct patterns of polarization during SCIRI. (A) Examples of flow cytometry analysis of the spinal cord are shown, identifying monocyte-derived macrophages (MDMs) and microglia as CD $45^{+} \mathrm{CDI} / \mathrm{b}^{\text {high }}$ and $\mathrm{CD} 45^{+} \mathrm{CDI} / \mathrm{b}^{\text {low }}$, respectively. (B) Spontaneous infiltration of circulating monocytes to the spinal cord increased after SCIRI. (C-F) Quantification of expression of CD86 (C and E), Dectin (D and F) in MDMs and microglia in spinal cord after SCIRI. Cells were pooled from 3 mice per group for a total of 3 separate experiments for each time point. $* P<0.05$, $* * P<0.01$ compared with the Sham group. Abbreviations: MDMs, monocyte-derived macrophages; SCIRI, spinal cord ischemia/reperfusion injury.

monocyte infiltration was not evident until day 3. The underlying mechanism that limited monocyte transmigration into re-perfused spinal cord tissue are yet unclear. Previous studies ${ }^{38}$ suggested that leukocytes migration into the CNS is highly regulated, requiring coordinated activation of the leukocytes and endothelium, together with appropriate chemoattractant gradients between blood and brain. Furthermore, we observed that invading MDMs decrease at day 7 after SCIRI, which was similar with other types of spinal cord injury, ${ }^{3,8,11}$ suggesting that the ablation of MDMs from the second week onward may have no effect on functional recovery. Taken together, these two observations indicated that the role of MDMs is essentially restricted to the first week after SCIRI, and probably only between days 3 and 7 post-injury.

Importantly, the present study highlights the fact that infiltrating MDMs and resident microglia differ in phenotype following SCIRI. Previous studies on SCIRI generally eliminated both these cells at the lesion site. ${ }^{39}$ However, MDMs may exert distinct functions from microglia. For example, substantial evidence has established that invading MDMs induce demyelination and neuron damage, whereas microglia protect neuron via clearing debris. Furthermore, the effect of monocyte infiltration is 


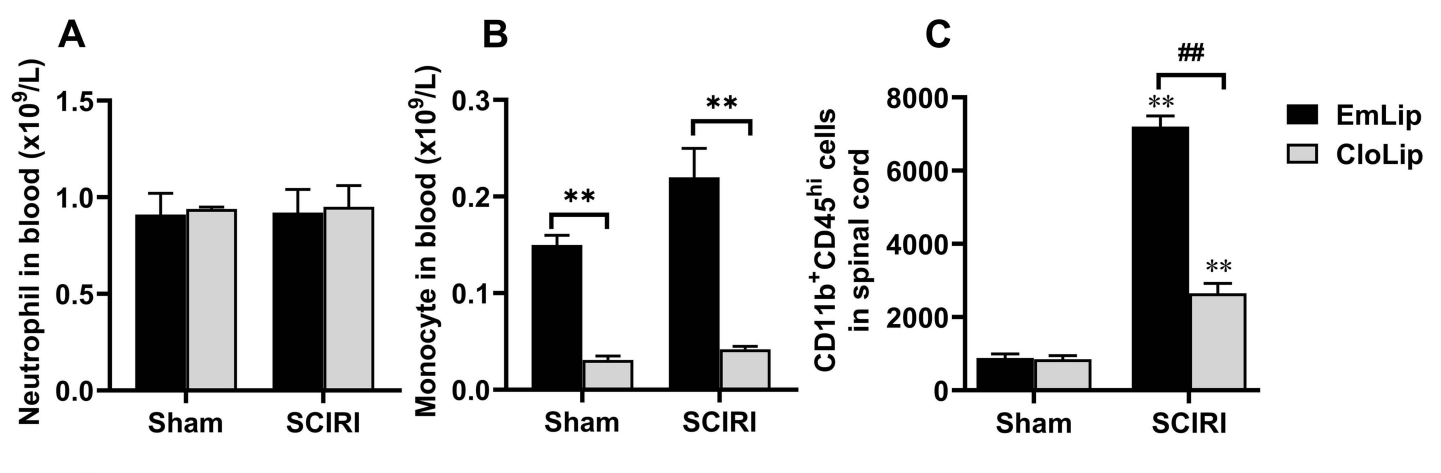

D

E
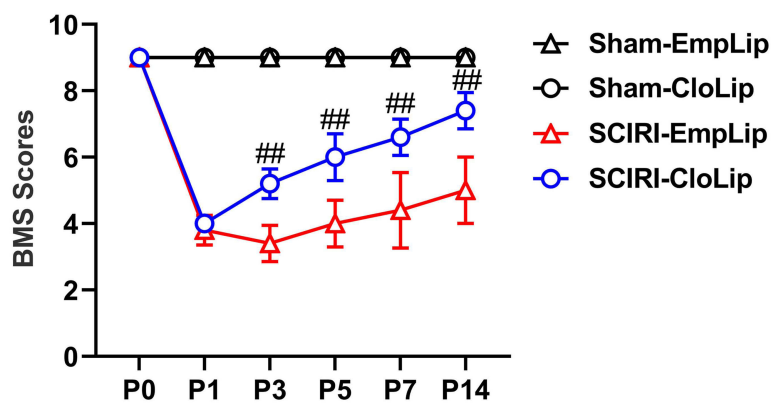

$\mathbf{F}$
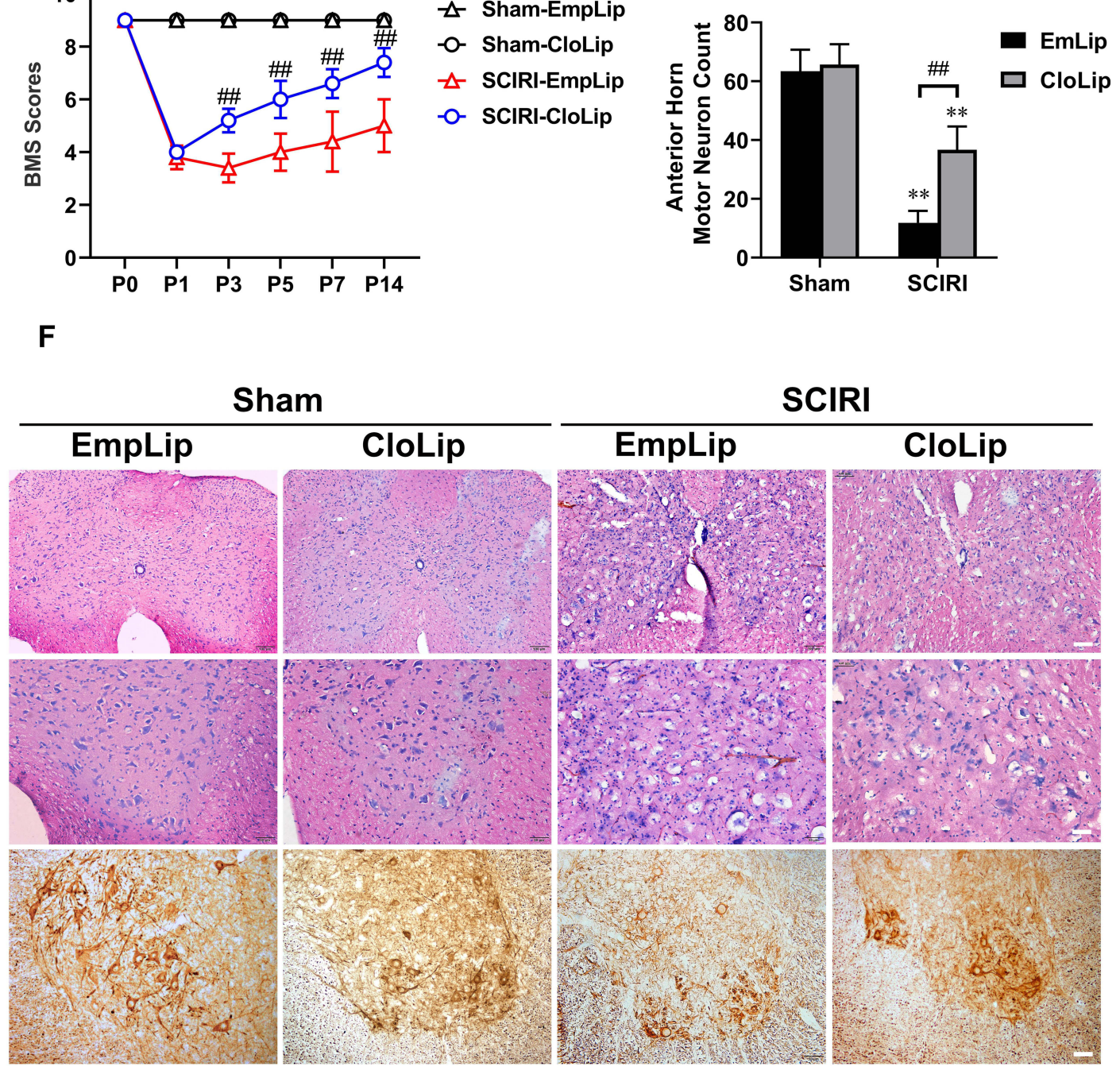

Figure 6 Depletion of MDMs improves long-term functional recovery and spinal cord damage after SCIRI. (A and B) Treatment with clodronate liposomes showed no effect on neutrophils but significantly reduced circulating monocytes in blood; (C) MDMs infiltration (recognized as CDI Ib ${ }^{+}$CD45 ${ }^{\text {hi }}$ cells) showed a marked elevation following SCIRI compared with the Sham group; While clodronate liposomes instead of empty liposomes application significantly alleviate MDMs infiltration at P3 following SCIRI; (D) Consecutive BMS scores showed a prominent elevation in SCIRI+CloLip group compared with the SCIRI+EmpLip group at P3, P5, P7 and PI4 following SCIRI; (E) SCIRI induced a significant motor neuron loss at the anterior horn both in the CloLip and EmpLip groups; While a marked elevation of motor neuron counting could be detected at day 3 after clodronate liposomes treatment; (F) HE staining and Immunohistochemistry for NF-200 in the spinal cord in each group at day 3 following SCIRI. the Ist line scale bar $=100 \mu \mathrm{m}$, line $2 \mathrm{nd}$ and $3 \mathrm{rd}$ scale bar $=25 \mu \mathrm{m}$. $\mathrm{n}=3 \mathrm{per}$ group at each time point. **Represented $P<0.01$, compared with the Sham group; ${ }^{\prime \prime}$ Represented $P<0.01$ compared with the SCIRI+CloLip group.

Abbreviations: MDMs, monocyte-derived macrophages; SCIRI, spinal cord ischemia/reperfusion injury. CloLip, clodronate-liposome; EmpLip, empty liposome. 
dependent on disease context. In EAE, infiltrating MDMs are highly inflammatory compared with microglia. But the data in traumatic spinal cord injury was inconsistent. Lee et $\mathrm{al}^{41}$ previously reported that depletion of the MDMs showed no effect on spontaneous improvement after traumatic spinal cord injury. But later, Shechter et $\mathrm{al}^{42}$ found that mice depleted of MDMs had a greater spread of damage in spinal cord lesion, revealing the neuroprotection of MDMs. In contrast, our present study attributed a pro-inflammatory function to MDMs primarily at an early stage following SCIRI; meanwhile, resident microglia contributed the main part of M2 phenotype at a later stage. Consistently, the alleviation of neurological function after MDMs depletion further confirmed their detrimental effects following SCIRI. These different findings between SCIRI and traumatic spinal cord injury might be explained by their distinct pathophysiology between traumatic and ischemic spinal cord injury. SCIRI occurs as a consequence of the two different stages of spinal cord injury. The first is ischemia caused by aortic transection or circulatory arrest, and then followed by reperfusion, leading to oxidative stress, inflammation and apoptosis. ${ }^{43}$

Notably, our previous study has reported an increased ratio of M1/M2-like monocytes in the peripheral circulation in patients with acute aortic dissection (AAD). ${ }^{44}$ The phenomenon indicates when AAD patients are subjected to deep hypothermic circulatory arrest, increased numbers of M1 polarized macrophages would migrate to the spinal cord after reperfusion, which may aggravate the severity of inflammation and subsequent neurological damages caused by SCIRI. Thus, timely targeting at infiltrating monocytes may help to reduce the clinical incidence of paraplegia occurring after thoracoabdominal aortic surgery.

\section{Conclusion}

Overall, our present study revealed differential roles of infiltrating MDMs and resident microglia following SCIRI. Our findings suggest that inflammatory MDMs worsen neurological outcome after murine SCIRI and may represent a therapeutic target for patients.

\section{Abbreviations}

MDMs, monocyte-derived macrophages; SCIRI, spinal cord ischemia/reperfusion injury; BMS, Basso mouse scale; CNS, central nervous system; DAC, descending aorta clamping; FMBP, femoral mean blood pressure; $\mathrm{HE}$, hematoxylin-eosin; BE, base excess; iNOS, inducible NO synthase; TNF- $\alpha$, tumor necrosis factor alpha; Arg-1, arginase-1; IL-4, interleukin 4; GAPDH, glyceraldehyde3-phosphate dehydrogenase; EAE, experimental autoimmune encephalomyelitis; AAD, acute aortic dissection.

\section{Author Contributions}

All authors made a significant contribution to the work reported, whether that is in the conception, study design, execution, acquisition of data, analysis and interpretation, or in all these areas; took part in drafting, revising or critically reviewing the article; gave final approval of the version to be published; have agreed on the journal to which the article has been submitted; and agree to be accountable for all aspects of the work.

\section{Funding}

This research was supported by the National Natural Science Foundation of China (NSFC 81873770 to $\mathrm{Li} \mathrm{H}$ and 81901231 to $\mathrm{Hu}$ ZL), the Foundation of Provincial Health Committee (HNA202101002 to Yang L), and the Fundamental Research Funds for the Central Universities of Central South University to Wang P.

\section{Disclosure}

The authors report no conflicts of interest in this work.

\section{References}

1. Greenberg RK, Lu Q, Roselli EE, et al. Contemporary analysis of descending thoracic and thoracoabdominal aneurysm repair: a comparison of endovascular and open techniques. Circulation. 2008;118(8):808-817. doi:10.1161/CIRCULATIONAHA.108.769695

2. Safi HJ, Miller III CC, Huynh TT, et al. Distal aortic perfusion and cerebrospinal fluid drainage for thoracoabdominal and descending thoracic aortic repair: ten years of organ protection. Ann Surg. 2003;238(3):372. doi:10.1097/01.sla.0000086664.90571.7a

3. Kong X, Gao J. Macrophage polarization: a key event in the secondary phase of acute spinal cord injury. J Cell Mol Med. 2017;21 (5):941-954. doi:10.1111/jcmm.13034

4. Wan S, Cheng Y, Jin H, et al. Microglia activation and polarization after intracerebral hemorrhage in mice: the role of protease-activated receptor-1. Transl Stroke Res. 2016;7(6):478-487. doi:10.1007/s12975-016-0472-8

5. Xiong X-Y, Liu L, Yang Q-W. Functions and mechanisms of microglia/macrophages in neuroinflammation and neurogenesis after stroke. Prog Neurobiol. 2016;142:23-44.

6. Francos-Quijorna I, Amo-Aparicio J, Martinez-Muriana A, LópezVales R. IL-4 drives microglia and macrophages toward a phenotype conducive for tissue repair and functional recovery after spinal cord injury. Glia. 2016;64(12):2079-2092. doi:10.1002/glia.23041

7. Durafourt BA, Moore CS, Zammit DA, et al. Comparison of polarization properties of human adult microglia and blood-derived macrophages. Glia. 2012;60(5):717-727. doi:10.1002/glia.22298

8. Girard S, Brough D, Lopez-Castejon G, Giles J, Rothwell NJ, Allan SM. Microglia and macrophages differentially modulate cell death after brain injury caused by oxygen-glucose deprivation in organotypic brain slices. Glia. 2013;61(5):813-824. doi:10.1002/ glia. 22478 
9. Yamasaki R, Lu H, Butovsky O, et al. Differential roles of microglia and monocytes in the inflamed central nervous system. J Exp Med. 2014;211(8):1533-1549. doi:10.1084/jem.20132477

10. Evans TA, Barkauskas DS, Myers JT, et al. High-resolution intravital imaging reveals that blood-derived macrophages but not resident microglia facilitate secondary axonal dieback in traumatic spinal cord injury. Exp Neurol. 2014;254:109-120. doi:10.1016/j. expneurol.2014.01.013

11. Feng L, Murugan M, Bosco DB, et al. Microglial proliferation and monocyte infiltration contribute to microgliosis following status epilepticus. Glia. 2019;67(8):1434-1448.

12. Hu X, Leak RK, Shi Y, et al. Microglial and macrophage polarization —new prospects for brain repair. Nat Rev Neurol. 2015;11(1):56-64. doi:10.1038/nrneurol.2014.207

13. Zhou X, Wahane S, Friedl MS, et al. Microglia and macrophages promote corralling, wound compaction and recovery after spinal cord injury via Plexin-B2. Nat Neurosci. 2020;23(3):337-350. doi:10.1038/s41593-020-0597-7

14. Milich LM, Ryan CB, Lee JK. The origin, fate, and contribution of macrophages to spinal cord injury pathology. Acta Neuropathol. 2019;137(5):785-797. doi:10.1007/s00401-019-01992-3

15. David S, Kroner A. Repertoire of microglial and macrophage responses after spinal cord injury. Nat Rev Neurosci. 2011;12 (7):388-399.

16. Awad H, Ankeny DP, Guan Z, Wei P, McTigue DM, Popovich PG. A mouse model of ischemic spinal cord injury with delayed paralysis caused by aortic cross-clamping. J Am Soc Anesthesiol. 2010;113 (4):880-891.

17. Das S, MacDonald K, Chang H-YS, Mitzner W. A simple method of mouse lung intubation. J Vis Exp. 2013;73. doi:10.3791/50318

18. Basso DM, Fisher LC, Anderson AJ, Jakeman LB, Mctigue DM, Popovich PG. Basso Mouse Scale for locomotion detects differences in recovery after spinal cord injury in five common mouse strains. $J$ Neurotrauma. 2006;23(5):635-659. doi:10.1089/neu.2006.23.635

19. He L, Xu J-M, Li H, et al. Moderate hypothermia increased the incidence of delayed paralysis through activation of the spinal microglia in an aortic cross-clamping rat model. Int $J$ Cardiol. 2016;220:454-461. doi:10.1016/j.ijcard.2016.06.169

20. Wang Z, Wu J-L, Zhong F, et al. Upregulation of proBDNF in the mesenteric lymph nodes in septic mice. Neurotox Res. 2019;36 (3):540-550. doi:10.1007/s12640-019-00081-3

21. Grant MC, Yang D, Wu CL, Makary MA, Wick EC. Impact of enhanced recovery after surgery and fast track surgery pathways on healthcare-associated infections: results from a systematic review and meta-analysis. Ann Surg. 2017;265(1):68-79. doi:10.1097/SLA.0 000000000001703

22. Gao T, Raza SA, Ramesha S, et al. Temporal profiling of Kv1. 3 channel expression in brain mononuclear phagocytes following ischemic stroke. $J$ Neuroinflammation. 2019;16(1):1-11. doi:10.1186/s12974-019-1510-8

23. Parney IF, Waldron JS, Parsa AT. Flow cytometry and in vitro analysis of human glioma-associated macrophages. $J$ Neurosurg. 2009;110(3):572-582. doi:10.3171/2008.7.JNS08475

24. Azambuja JH, Schuh RS, Michels LR, et al. Blockade of CD73 delays glioblastoma growth by modulating the immune environment. Cancer Immunol Immunother. 2020;69(9):1801-1812. doi:10.1007/s00262-020-02569-w

25. Kumar A, Alvarez-Croda D-M, Stoica BA, Faden AI, Loane DJ. Microglial/macrophage polarization dynamics following traumatic brain injury. J Neurotrauma. 2016;33(19):1732-1750. doi:10.1089/ neu.2015.4268

26. Denker SP, Ji S, Dingman A, et al. Macrophages are comprised of resident brain microglia not infiltrating peripheral monocytes acutely after neonatal stroke. J Neurochem. 2007;100(4):893-904. doi:10.1 111/j.1471-4159.2006.04162.x
27. Xin D, Li T, Chu X, et al. Mesenchymal stromal cell-derived extracellular vesicles modulate microglia/macrophage polarization and protect the brain against hypoxia-ischemic injury in neonatal mice by targeting delivery of miR-21a-5p. Acta Biomater. 2020;113:597-613. doi:10.1016/j.actbio.2020.06.037

28. Sousa C, Golebiewska A, Poovathingal SK, et al. Single-cell transcriptomics reveals distinct inflammation-induced microglia signatures. EMBO Rep. 2018;19(11):e46171. doi:10.15252/ embr.201846171

29. Hsieh CL, Kim CC, Ryba BE, et al. Traumatic brain injury induces macrophage subsets in the brain. Eur $J$ Immunol. 2013;43 (8):2010-2022. doi:10.1002/eji.201243084

30. Kigerl KA, Gensel JC, Ankeny DP, Alexander JK, Donnelly DJ, Popovich PG. Identification of two distinct macrophage subsets with divergent effects causing either neurotoxicity or regeneration in the injured mouse spinal cord. $J$ Neurosci. 2009;29 (43):13435-13444. doi:10.1523/JNEUROSCI.3257-09.2009

31. Ma S-F, Chen Y-J, Zhang J-X, et al. Adoptive transfer of M2 macrophages promotes locomotor recovery in adult rats after spinal cord injury. Brain Behav Immun. 2015;45:157-170. doi:10.1016/j. bbi.2014.11.007

32. Chawla A. Control of macrophage activation and function by PPARs. Circ Res. 2010;106(10):1559-1569. doi:10.1161/CIRCRESA HA. 110.216523

33. Murray PJ, Wynn TA. Obstacles and opportunities for understanding macrophage polarization. J Leukoc Biol. 2011;89(4):557-563. doi:10.1189/jlb.0710409

34. Hao J, Hu Y, Li Y, Zhou Q, Lv X. Involvement of JNK signaling in IL4-induced M2 macrophage polarization. Exp Cell Res. 2017;357 (2):155-162. doi:10.1016/j.yexcr.2017.05.010

35. Orr MB, Gensel JC. Spinal cord injury scarring and inflammation: therapies targeting glial and inflammatory responses. Neurotherapeutics. 2018;15(3):541-553. doi:10.1007/s13311-0180631-6

36. Akaishi T, Takahashi T, Nakashima I. Peripheral blood monocyte count at onset may affect the prognosis in multiple sclerosis. $J$ Neuroimmunol. 2018;319:37-40. doi:10.1016/j.jneuroim.2018.0 3.016

37. Zarruk JG, Greenhalgh AD, David S. Microglia and macrophages differ in their inflammatory profile after permanent brain ischemia. Exp Neurol. 2018;301:120-132. doi:10.1016/j.expneurol.2017.08.011

38. Ransohoff RM, Kivisäkk P, Kidd G. Three or more routes for leukocyte migration into the central nervous system. Nat Rev Immunol. 2003;3(7):569-581. doi:10.1038/nri1130

39. Franco R, Fernandez-Suarez D. Alternatively activated microglia and macrophages in the central nervous system. Prog Neurobiol. 2015;131:65-86.

40. Ajami B, Bennett JL, Krieger C, McNagny KM, Rossi FM. Infiltrating monocytes trigger EAE progression, but do not contribute to the resident microglia pool. Nat Neurosci. 2011;14(9):1142-1149. doi: $10.1038 / \mathrm{nn} .2887$

41. Lee SM, Rosen S, Weinstein P, van Rooijen N, Noble-Haeusslein LJ. Prevention of both neutrophil and monocyte recruitment promotes recovery after spinal cord injury. $J$ Neurotrauma. 2011;28 (9):1893-1907. doi:10.1089/neu.2011.1860

42. Shechter R, London A, Varol C, et al. Infiltrating blood-derived macrophages are vital cells playing an anti-inflammatory role in recovery from spinal cord injury in mice. PLoS Med. 2009;6(7): e1000113. doi:10.1371/journal.pmed.1000113

43. Ghincea CV, Ikeno Y, Aftab M, Reece TB Spinal cord protection for thoracic aortic surgery: bench to bedside. Paper presented at: Seminars in thoracic and cardiovascular surgery; 2019.

44. Shen WY, Luo C, Reinaldo Hurtado P, et al. The regulatory role of ProBDNF in monocyte function: implications in Stanford type-a aortic dissection disease. FASEB J. 2020;34(2):2541-2553. doi:10.1096/fj.201901905RR 


\section{Publish your work in this journal}

The Journal of Inflammation Research is an international, peerreviewed open-access journal that welcomes laboratory and clinical findings on the molecular basis, cell biology and pharmacology of inflammation including original research, reviews, symposium reports, hypothesis formation and commentaries on: acute/chronic inflammation; mediators of inflammation; cellular processes; molecular mechanisms; pharmacology and novel anti-inflammatory drugs; clinical conditions involving inflammation. The manuscript management system is completely online and includes a very quick and fair peerreview system. Visit http://www.dovepress.com/testimonials.php to read real quotes from published authors. 